International Journal of Modern Physics E

(C) World Scientific Publishing Company

\title{
WHAT EINSTEIN DID NOT KNOW
}

\author{
MARTIN L. PERL \\ Stanford Linear Accelerator Center, Stanford University \\ 2575 Sand Hill Road, Menlo Park, CA 94025, U.S.A. \\ martin@slac.stanford.edu
}

\begin{abstract}
This public lecture is about 100 years of research on elementary particles and fundamental forces, beginning with the identification of the electron about 1900 and extending to the astonishing discovery of Dark Matter in the late 1900s. I talk about the elementary particle concept; the discoveries of leptons, quarks and force carrying particles; and some of the experimental technology used. I tell of my own research, our discovery of the tau lepton, our long, inconclusive search for fractional charged particles, and my new involvement in astronomical research on Dark Matter. I conclude by looking ahead to old unsolved puzzles and new questions on the fundamental nature of matter and force that face us in the 21st Century.
\end{abstract}

\section{Introduction: What Einstein Did Not Know}

I gave this public lecture at the Symposium on 50+ Years of High Energy Physics at the University of Buffalo in honor of Professor Lal Jain, a great pioneer in high energy physics.

The year 2005 was celebrated as the hundredth anniversary of Albert Einstein's great creative year of 1905 . This was an important celebration but I feel that it was misleading. Many people may not have realized that since the time of Einstein's major work, 1900 through the 1920s, tremendous new understanding has been acquired about elementary particles, fundamental forces, astrophysics and cosmology. Einstein died in 1955 , but in his published work he mostly ignored the new experimental and theoretical understanding of the 1930s and 1940s: the discovery of the neutron, the discovery of the muon, the elucidation of the nuclear force, Wolfgang Pauli's deduction of the existence of the neutrino, and the early understanding of the weak force. Einstein's long and profound effort to devise a unified field theory was concerned with just the gravitational and electromagnetic forces, he ignored the nuclear and weak force. No matter how great the contributions to physics of any person, physics always moves on. In this public lecture I summarize this new understanding. I also talk about the puzzles we have not yet solved and the questions we have not yet answered; some of these puzzles and questions are new but some are old.

This lecture has nine short sections: 


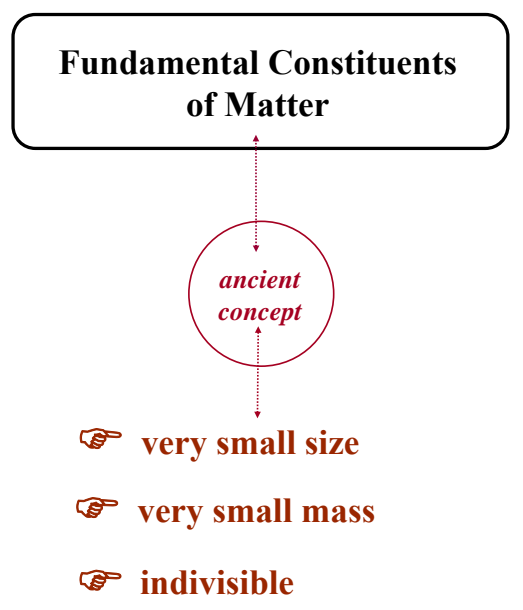

Fig. 1. The idea of an elementary particle.

- 1. Introduction: What Einstein Did Not Know

- 2. Elementary Constituents of Matter and Fundamental Forces

- 3. The Leptons

- 4. The Quarks

- 5. The Force Carrying Particles and the Standard Model of Particle Physics

- 6. Dark Matter

- 7. Not All Experiments Are Fruitful: Our Search for Fractional Charge Particles

- 8. A Change of Research Technology and a New Telescope

- 9. Old Unsolved Puzzles and New Questions in the 21st Century

\section{Elementary Constituents of Matter and Fundamental Forces}

\subsection{From living things and everyday objects to elementary particle}

The concept of an elementary particle, Fig. 1, is an ancient concept. The known elementary particles are very much smaller than living things and everyday objects. It is necessary to use the notation $10^{n}$ to give the size of these particles in terms of centimeters. (There are 2.5 centimeters in an inch .) $10^{n}$ means a number consisting of a 1 followed by $\mathrm{n}$ zeros. For example $10^{5}=100,000$. Also $10^{-n}$ means a fraction with 1 in the numerator and 1 followed by $n$ zeros in the denominator. Thus $10^{-5}=$ $1 / 100,000$. 
Figures 2 and 3 show how the matter in living things and everyday objects is made up of smaller and smaller constituents, finally being composed of elementary particles - electrons and quarks. There are no reliable experiments or observations in which the electron or quark has been further divided. The discovery history is also shown.

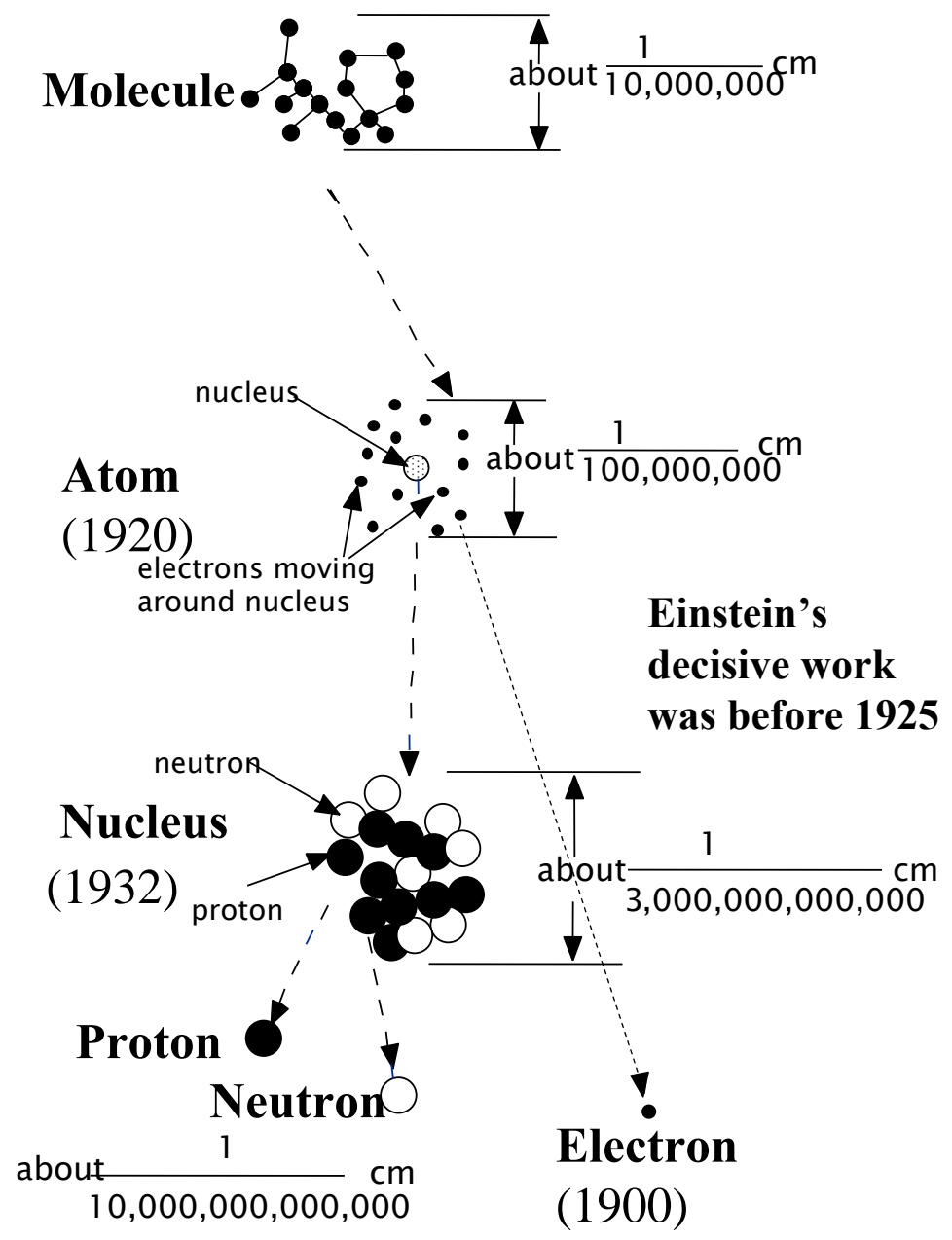

Fig. 2. The chemical, biological and structural properties of matter depend the type of molecules and atoms. The molecules and atoms are composed of much smaller particles, including the electron elementary particle. 


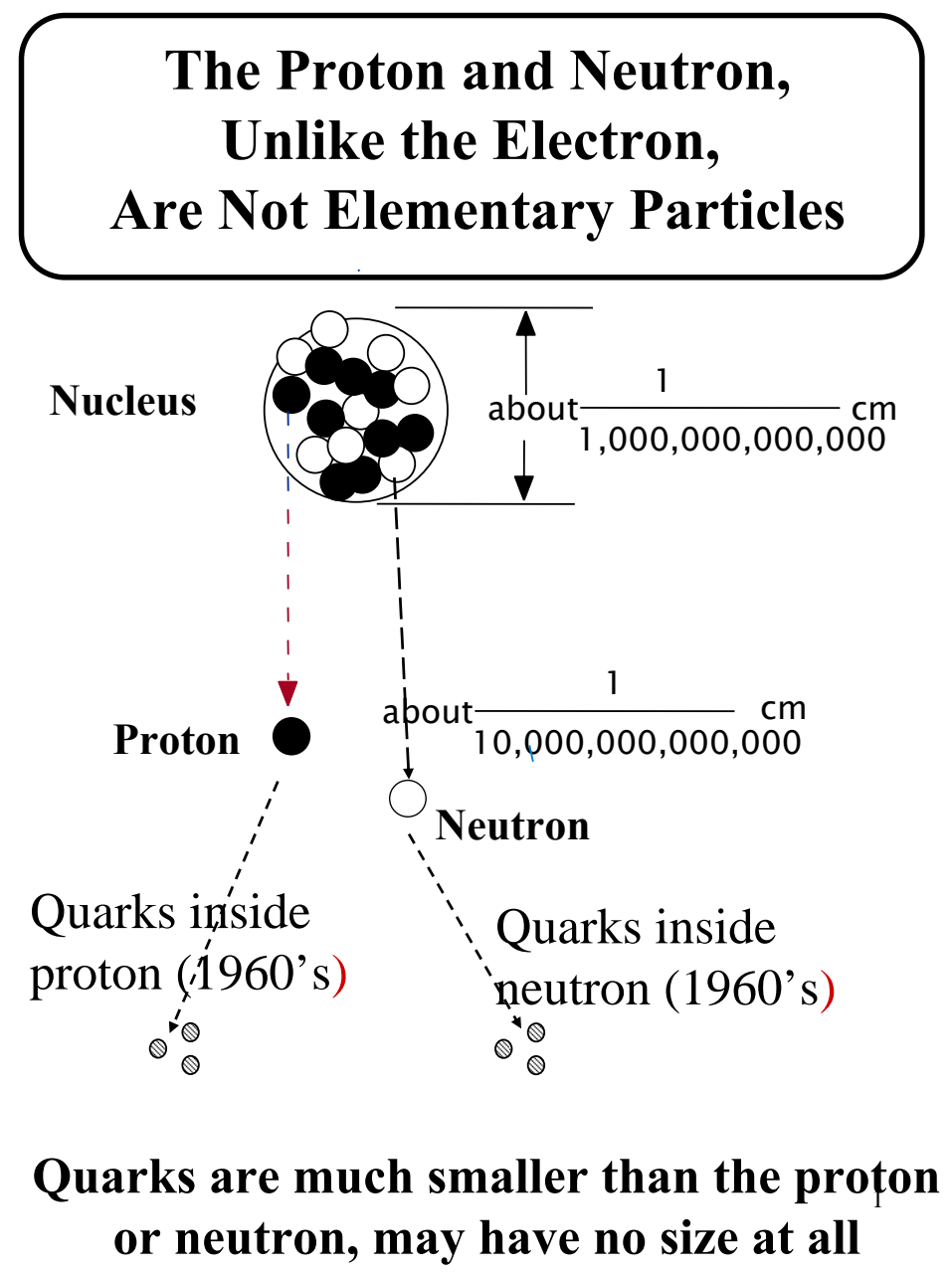

Fig. 3. The proton and neutron are small particles but not elementary, they are composed of the quark elementary particles.

\subsection{The electron, identified about one hundred years ago}

The electron was the first elementary particle to be discovered, identified and its nature clarified about the year 1900, after about 30 years of experiments. Its mass, electric charge and size are extremely small compared to everyday objects

- The electron mass is about $10^{-27}$ grams (there are 454 grams in a pound).

- The charge of the electron is also very small, roughly $10^{-19}$ coulombs. A fully charged automotive storage battery contains about $10^{6}$ coulombs. The electron charge is negative. 
- If we think of the electron as a classical particle its size is less than about $10^{-16}$ centimeters. However in quantum mechanics a particle can also be considered a wave and then the size concept does not apply.

- All experiments attempting to break up the electron have failed. An electron can be made to disappear by annihilating it with its positively charged partner, called a positron, but no one has succeeded in separating an electron into two or more parts. Therefore at present the electron is taken to be indivisible

The electron's charge, size and indivisibility are representative of the properties of the other elementary particles. However the masses of the elementary particles vary widely.

- The ratio of the photon mass to the electron mass is less than about $10^{-22}$. All measurements are consistent with the photon having zero mass.

- The most massive known particle, the top quark, has about $3 \times 10^{5}$ the electron mass.

\subsection{Puzzles about the nature of the electron}

Although the electron has been intensively studies for a hundred years there are old puzzles about its nature that have still not be solved:

- No one knows how to calculate the mass or the charge of the electron from first principles. In the accepted theory of the electron the measured mass and charge are simply inserted.

- We do not know if the electron has any size at all. Perhaps it is just a point surrounded by a field of forces?

\subsection{The four fundamental forces}

By the middle of the 20th Century four fundamental forces had been discovered. These forces act on some or all of the elementary particles:

- Gravitational force: the first force recognized. Its theory was first quantified by Newton in the late 1600s and then extended by Einstein to general relativity in the 1910s and 1920s.

- Electromagnetic force: its theory was quantified in the 19th Century by Maxwell.

- Nuclear force: it holds the nucleus together and is also called the strong force. The basic theory was understood by about 1940 .

- Weak force: it is the cause of most radioactivity. Weak force theory was mostly understood by 1970. Einstein's work on developing a unified theory of forces was concerned just with the gravitational and electromagnetic forces. It is strange that he was never deeply concerned with the nuclear and weak forces. Contrary to Einstein's hope the presently accepted unified theory of forces connects the 
electromagnetic, weak and strong forces but there is no agreement on how to incorporate the gravitational force.

\subsection{Aside on the proton and neutron}

The proton and neutron, Figs. 2 and 3, are not elementary particles, they are composite particles made up of quarks, The attractive nuclear force between the quarks holds the neutron and proton together.

- Size: the size of the proton and the neutron is about $10^{-13}$ centimeters, thus unlike the electron and other elementary particles the proton and neutron are not point particles, but have a clearly measurable size. Since quarks are much smaller, less than $10^{-16}$ centimeters, the proton and neutron are mostly empty space.

- Charge: the proton has the same magnitude of charge as the electron, but the proton has positive charge while the electron has negative charge.

- Mass: the proton's mass is 1840 times the electron mass, while the neutron is slightly heavier, with a mass 1842 times the electron mass.

- Stability: the proton is stable but the free neutron is unstable, decaying into a proton and other particles.

\section{The Leptons}

\subsection{The electron and the lepton family of elementary particles}

The electron is acted upon by the gravitational, electromagnetic and weak forces but not by the nuclear force. And there are five other elementary particles, listed in Table 1, called leptons, that are acted upon by the same set of forces. In Table 1 and all other tables I give particle masses in units of the electron mass. There is no significance to using the electron mass as a unit, it is just convenient. We understand very little about mass and we do not know a fundamental mass unit.

The muon and the tau have the same electric charge as the electron, but they are more massive than the electron and are unstable. There are three more leptons with zero electric charge, called neutrinos, associated with the electron, muon, and tau.

\subsection{The muon}

The muon was discovered in cosmic rays in the first half of last century, Fig. 4. It mostly decays into an electron and two neutrinos, Fig. 5 .

\subsection{The tau}

My colleagues and I discovered the tau lepton in the 1970s. While still at the University of Michigan in the early 1960s, I thought a lot about the relation of the muon 
Table 1. The six known leptons. The charged leptons: electron, muon and tau have the same size charge; their associated neutrinos have zero charge. The masses of the electron, muon and tau have been precisely measured, the ratio of their masses is more precisely known than indicated in the table. It is difficult to directly measure the masses of the neutrinos, hence the table gives upper limits. But the there is an upper limit on the sum of the neutrino masses derived from astronomical observations and astrophysics. The upper limit on the sum of the neutrino masses relative to the electron mass is abut $2 \times 10^{-6}$. Therefore the neutrinos all have small masses relative to the electron, but from the behavior of neutrinos we know that their masses are not zero.

\begin{tabular}{ccc}
\hline Lepton name & Mass relative to electron mass & Magnitude of charge relative to electron charge \\
\hline Electron & 1 & 1 \\
Electron-neutrino & $<4 \times 10^{-6}$ & 0 \\
Muon & 207 & 1 \\
Muon-neutrino & $<0.33$ & 0 \\
Tau & 3480 & 1 \\
Tau-neutrino & $<30$ & 0 \\
\hline
\end{tabular}

to the electron. Why is the muon mass about 200 times the electron mass although both particles are subject to the same three forces: gravitational, electromagnetic and weak? In 1963 I joined the Stanford Linear Accelerator Center of Stanford University, the linear accelerator at the Center produces primary electron beams and secondary muon beams. My idea was to study muon interactions to elucidate the electron-muon difference, but I learned nothing fundamental about the relation of the muon to electron in seven year of experimentation.

About 1970, I then developed the sequential lepton hypothesis in which I predicted that there is a sequence of charged leptons, heavier and heavier, each with its own neutrino, Table 2 . I used the symbols $L_{1}, L_{2} \ldots$ to denote the heavier members of the sequence.

Table 2. The first four members of my sequential lepton hypothesis before my discovery of the tau.

\begin{tabular}{ccc}
\hline Lepton name & Mass relative to electron mass & Charge relative to electron charge \\
\hline Electron & 1 & 1 \\
Electron-neutrino & see Table 1 & 0 \\
Muon & 207 & 1 \\
Muon-neutrino & see Table 1 & 0 \\
& $>$ muon mass & 1 \\
$L_{1}$ & $?$ & 0 \\
$L_{1}$-neutrino & $>L_{1}$ mass & \\
$L_{2}$ & $?$ & 0 \\
$L_{2}$-neutrino & & \\
\hline
\end{tabular}




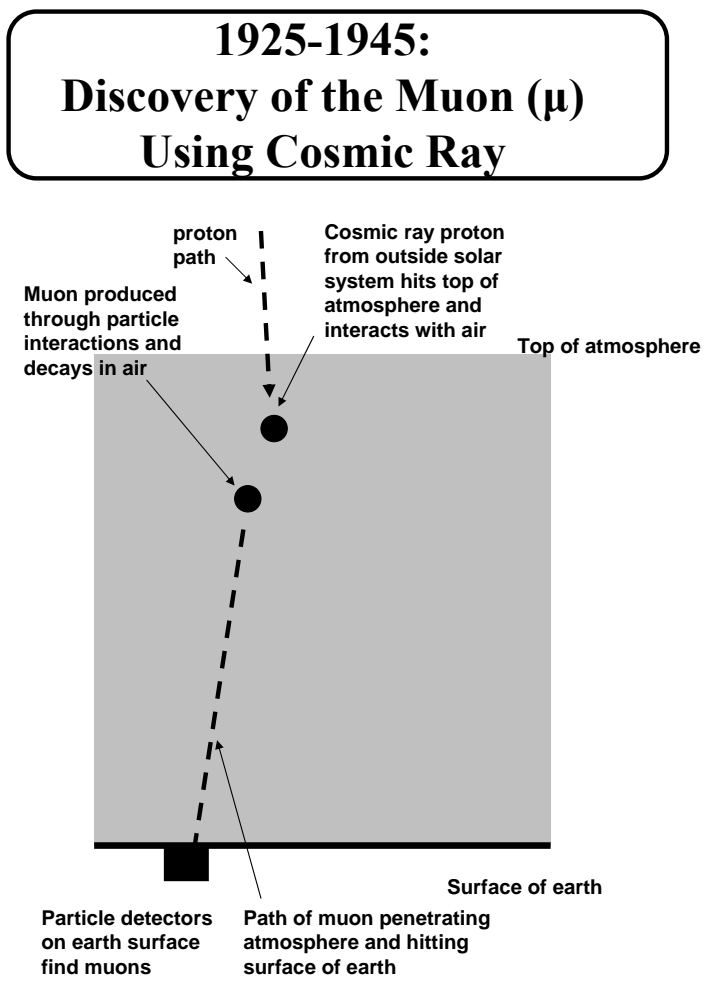

Fig. 4. Muons are usually produced when a high energy cosmic ray proton hits the earth's atmosphere. This was how they were discovered and their properties deduced in the 1930s and early 1940s. Since about 1950 muon research uses muons produced using particle accelerators.

In the late 1960s physicists at the Stanford Linear Accelerator Center and the Lawrence Berkeley Laboratory began to build an electron-positron colliding beams machine in which high energy electrons and positrons collided, Fig. 6.

It was expected from measurements at electron-positron collider already operating that in our SPEAR colliding beams machine the following reactions would occur:

$$
\begin{gathered}
\text { electron }+ \text { positron } \longrightarrow \text { electron }+ \text { positron } \\
\text { electron }+ \text { positron } \longrightarrow \text { negative }- \text { muon }+ \text { positive }- \text { muon }
\end{gathered}
$$

I dreamed that in this electron-positron colliding beams machine we could also produce the $L_{1}$ charged sequential lepton by the reaction:

$$
\text { electron }+ \text { positron } \longrightarrow \text { negative }-L_{1}+\text { positive }-L_{1}
$$




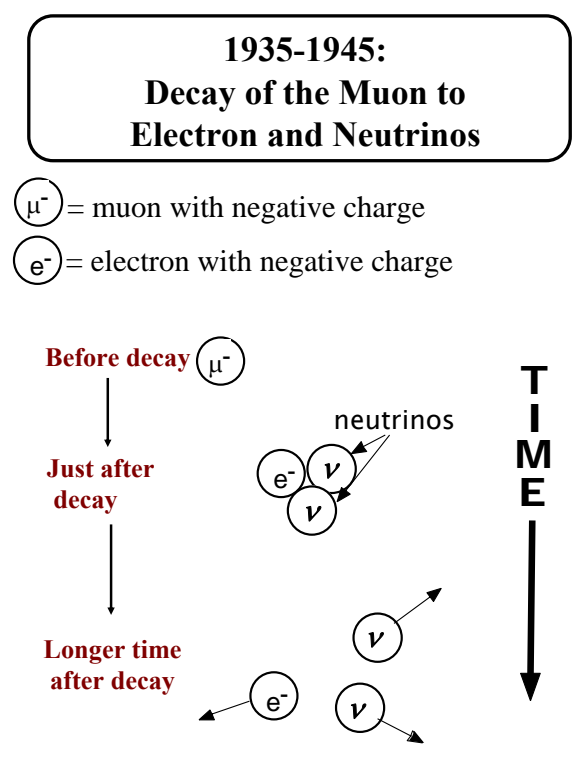

Fig. 5. A muon decays spontaneously in about $10^{-6}$ seconds usually into an electron and two neutrinos..

SPEAR began to run in 1974 and by 1975 I was seeing the reaction in Eq. 3. This new charged lepton, $L_{1}$, had a mass about 3500 times the electron mass so that it was much heavier than the electron and heavier than the muon. For about four years there was considerable skepticism in the particle physics community as to whether my interpretation of a new heavy, charged lepton was right. It was a hard time. But we kept finding this reaction and eventually other colliding beam experiments found the reaction. We named this new charged lepton with the Greek letter tau on the suggestion of Petros Lapidus since the Greek word $\tau \rho \iota \tau o \nu$ signifies three and the tau was the third charged lepton to be discovered. Thus we had discovered the special case of the reaction in Eq. 3:

$$
\text { electron }+ \text { positron } \longrightarrow \text { negative }- \text { tau }+ \text { positive }- \text { tau }
$$

In 1995 I received the Nobel Prize in Physics for the discovery of the tau.

\subsection{Another puzzle from the 20th Century: why are there just three charge leptons}

With the discovery of the tau, I and many other particle physicists expected that yet heavier charged leptons existed. Searches for additional charged leptons have been conducted at 50 times the energy at which the tau was discovered, but no additional charged leptons have been found. The null result of these searches and 


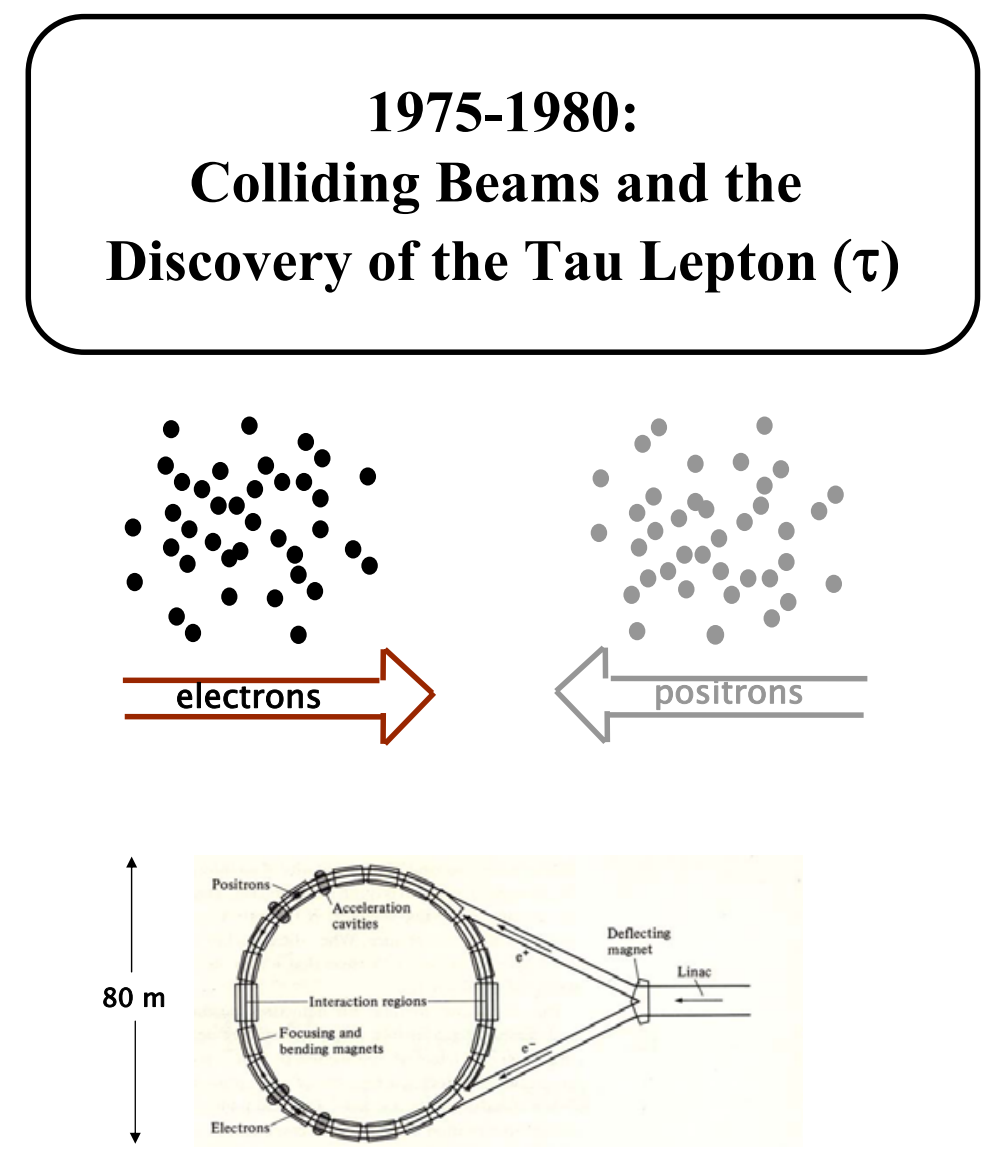

Fig. 6. Above: the principle of an electron-positron colliding beams machine. Below: the SPEAR electron-positron collider built at SLAC by physicists and engineers from SLAC and the Lawrence Berkeley Laboratory in the late 1960s and early 1970s

indirect evidence leads to the conclusion that just three types of charged leptons exist. Similarly only three types of neutrinos associated with these leptons exist. In the next section I note that there are just three pairs of quarks. We do not know why the number three is special, why not two or five? 


\section{Electron-Positron Annihilation into a $\mathrm{Tau}^{+}$and a Tau-}

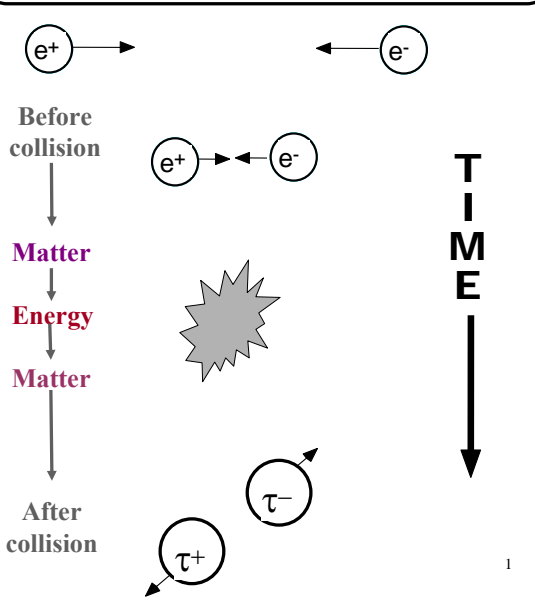

Fig. 7. The most straightforward way to produce tau leptons is through the collision and annihilation of an electron and a positron. The annihilation results in an unstable bundle of energy that turns into a pair of taus.

\section{The Quarks}

Protons and neutrons are composed of the elementary particles called quarks, Table 3. The behavior of quarks is more complicated than the behavior of leptons because the quarks interact through the nuclear force as well as through the gravitational, electromagnetic and weak forces. Unlike the leptons that can be isolated and studied separately, experimenters have not been able to isolate single quarks. For example, experimenters have not been able to break up the proton or neutron so that single quarks are produced, instead more protons or neutrons and other composite particles called mesons are produced.

\section{The Force Carrying Particles and the Standard Model of Particle Physics}

\subsection{The Force Carrying Particles}

There is yet one more family of elementary particles, the force carrying particles, Table 4. Relativistic quantum mechanics includes the basic principle that a fundamental force is carried by an elementary particle. The photon carries the electromagnetic force, the $\mathrm{Z}$ and $\mathrm{W}$ carry the weak force and the gluon carries the nuclear force. The existence and properties of the photon, Z, W, and gluon have been ex- 
Table 3. The six known quarks. Quarks have never been isolated, hence their mass cannot be measured directly and there are theoretical problem in interpreting from indirect measurements the mass of the up, down and strange quarks.

\begin{tabular}{ccc}
\hline Quark name & Mass relative to electron mass & Magnitude of charge relative to electron charge \\
\hline Up & 2.9 to 5.8 & $2 / 3$ \\
Down & 5.8 to 14 & $1 / 3$ \\
& & $2 / 3$ \\
Charm & $2.5 \times 10^{3}$ & $1 / 3$ \\
Strange & 140 to 240 & $2 / 3$ \\
Top & $3.4 \times 10^{5}$ & $1 / 3$ \\
Bottom & $9 \times 10^{3}$ & \\
\hline
\end{tabular}

tensively established and measured. On the contrary, there is no experimental or observational evidence that the gravitational force is carried by a particle. However it is usually assumed that there is such a particle and it is called the graviton. A few physicists doubt the existence of the graviton based on arguments that there is no fundamental way to combine relativistic quantum mechanics and general relativity.

Table 4. The five known force carrying particles. The electromagnetic force is carried by the photon, while the weak force is carried by two particles, the $\mathrm{Z}$ and the $\mathrm{W}$. There are several types of gluons that carry the nuclear force. The existence and properties of the photon, $\mathrm{Z}, \mathrm{W}$, and gluons have been established by experiment. It is a hypothetical concept that gravity is carried by a particle called a graviton, but there is no evidence for this concept, it is based on the assumption that gravity can be treated quantum mechanically.

\begin{tabular}{cccc}
\hline Force & Particle & Mass relative to electron & Magnitude of charge relative to electron charge \\
\hline Electromagnetic & Photon & $<10^{-22}$ & 0 \\
Weak & $\mathrm{Z}$ & $1.8 \times 10^{5}$ & 0 \\
Weak & $\mathrm{W}$ & $1.6 \times 10^{5}$ & 1 \\
Nuclear & gluon & 0 or close to 0 & 0 \\
Gravity & graviton & assumed to be 0 & 0 \\
\hline
\end{tabular}

\subsection{The limitations of the so-called Standard Model of particle physics}

Thus in the 20th Century we have learned a tremendous amount about elementary particles and fundamental forces. We know a tremendous amount about the masses, charges and other properties of sixteen elementary particles. (In this counting the various gluon types are counted as one gluon and the hypothetical graviton is excluded.) We know some relations between the particles and we know how to calculate the interactions of these particles. This body of experimental knowledge and consistent theory is called the Standard Model of particle physics, a great accomplishment. 
But the Standard Model is not a fundamental explanation of the world of elementary particles, it does not provide a way to calculate most of the properties of the particles from first principles, it does not provide a way to calculate the forces from first principles, it does not explain why there are $2 \times 3$ leptons and $2 \times 3$ quarks but 4 forces.

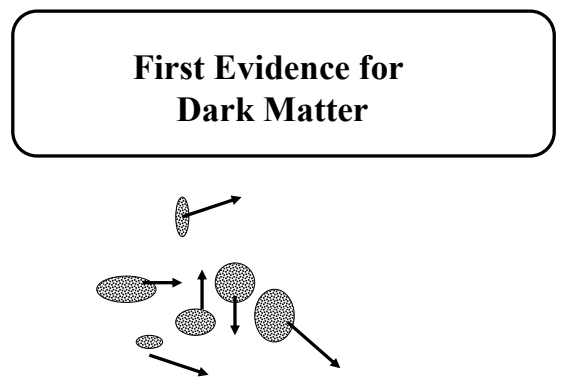

Zwicky in the 1930s showed that the velocities of galaxies in a cluster would cause the cluster to fall apart unless the galaxies had more mass than the luminous mass. Hence dark matter.

But strangely almost all physicists and most astronomers ignored Dark Matter for 30 years?

Fig. 8. First observations in the 1930s on the existence of Dark Matter.

\section{Dark Matter}

\subsection{The astonishing yet neglected discovery of Dark Matter}

All the experimental knowledge contained in the Standard Model was obtained using laboratory experiments, radioactivity, cosmic rays, and accelerators. Yet the very important discovery of Dark Matter was made in the 1930s, initially by Fritz Zwicky, using astronomical observations, Fig. 8.

Until then physicists and astronomers assumed that all the matter in the universe was similar to the material on earth and that this matter contained molecules made up of atoms, atoms consisting of electrons orbiting around a nucleus, ionized atoms, protons, electrons and a scattering of other charged particles. This matter can absorb and emit electromagnetic waves, such as light. The property of being able to absorb and emit electromagnetic waves is called the Luminous property of matter. Stars that shine and hot gases are obviously Luminous. Dead stars, planets, 
dust and cold gases become Luminous if heated or otherwise excited.

With the assumption that all matter is Luminous, the total matter in a galaxy used to be estimated by measuring the amount of light emitted by the galaxy. Then the average motion of galaxies in a cluster of galaxies can be calculated using the observed velocities of the galaxies and the gravitational attraction between the galaxies. In turn the gravitational attraction depends upon the galaxies masses. Zwicky observed that the average motion of the galaxies required larger galaxy masses, hence he proposed the term Dark Matter for additional matter that was not Luminous.

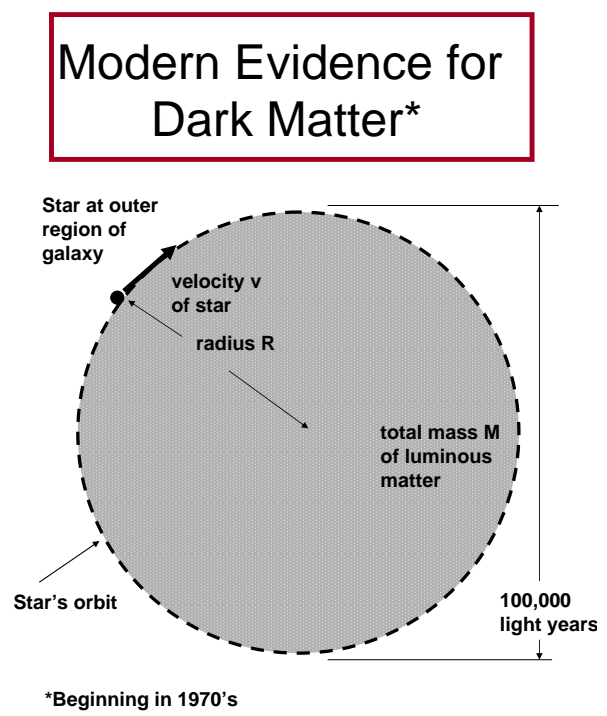

Fig. 9. Modern evidence for the existence of dark matter.

\subsection{The acceptance of the existence of Dark Matter}

Until the 1970s most physicists and some astronomers ignored the probable existence of Dark Matter. I cannot explain this ignorance, as I was one of the ignorant ones. Finally beginning in the 1970s more quantitative evidence for the existence of Dark Matter was developed. For example, Fig. 9, the velocity of stars with orbits in the outer reaches of a galaxy is larger than can be explained by the Luminous Mass of the galaxy, the presence of Dark Matter is required. In fact 5 or 6 times more Dark Matter than Luminous Matter is required and the Dark Matter is more spread out than the Luminous Matter in the galaxy. 


\subsection{What is Dark Matter}

At present all our knowledge of Dark Matter comes from astronomical observations and related astrophysical calculations. The present assumption is that Dark Matter consists of neutral elementary particles of unknown mass and perhaps exotic properties. The forces acting on the Dark Matter particle are as follows:

- The principle force is gravitation. At present most astronomer feel that all observations of Dark Matter are explained by just the gravitational force.

- Dark Matter particles may act through the weak force.

- Dark Matter particles are neutral, therefore they do not act through the electromagnetic force.

- Dark Matter particles do not act through the nuclear force.

One hypothesis is that the mass is in the range of several hundred to several thousand times the proton mass. Another hypothesis is that the mass is very small, much less than an electron mass. There is no experimental or observational proof of either hypothesis. Perhaps this is all wrong and Dark Matter does not consist of particles but is a mysterious fluid.

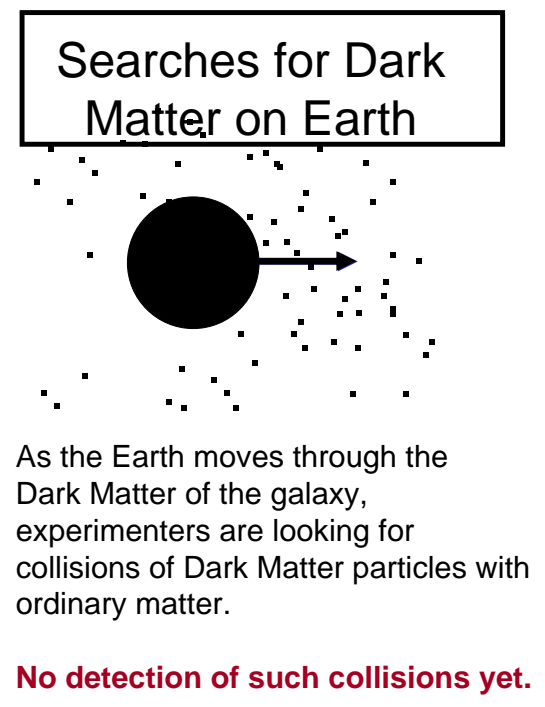

Fig. 10. Searching for Dark Matter as the earth moves through the Dark Matter cloud that fills the Galaxy. 


\subsection{Terrestrial searches for Dark Matter}

There are two ways to search for Dark Matter on the earth. One way is to try to produce Dark Matter particles in very high energy particle colliding beam machines. So far there is no evidence, many physicists hope these particles will be discovered at the CERN proton-proton colliding beam machine, now being constructed in Switzerland.

Figure 10 illustrates another search method in which it is hoped that as the earth moves through the cloud of Dark Matter particles in our galaxy, the collision of Dark Matter particles with nuclei can be detected. There are many experiments using this search method, but so far there is no success. These experiments are difficult because the absence of the electromagnetic and nuclear force leads to collisions between Dark Matter and nuclei being very rare. And if Dark Matter particles do not interact through the weak force, collisions through the remaining gravitational force are not detectable by present technology.

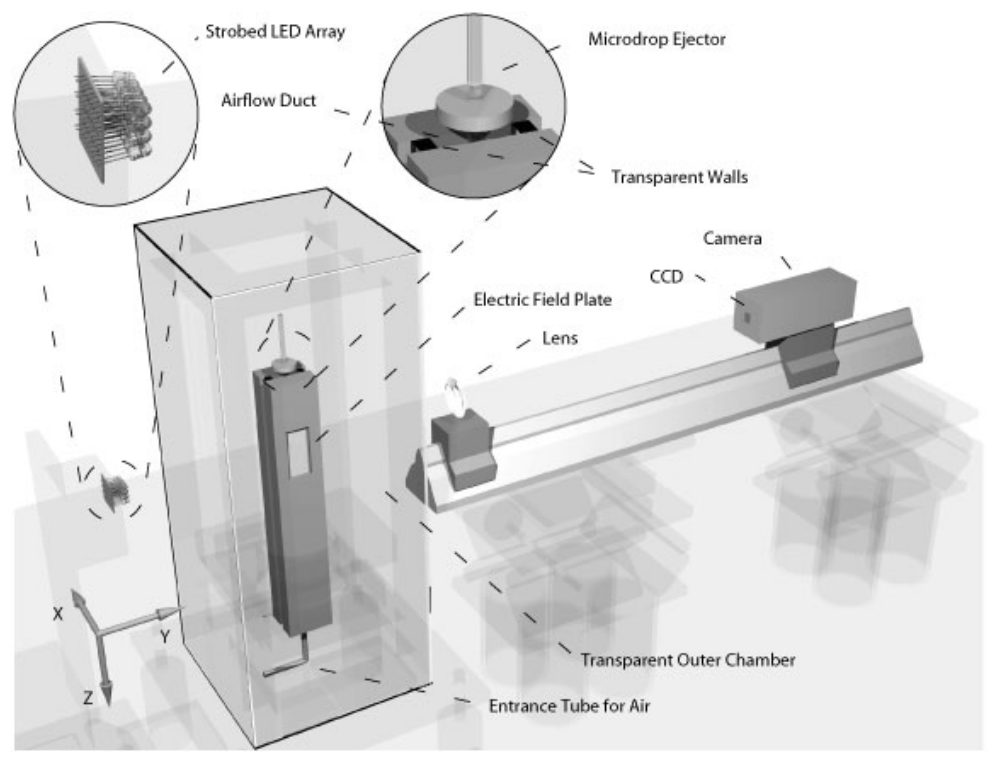

Fig. 11. Sketch of the apparatus we used to search for fractional charge particles.

\section{Not All Experiments Are Fruitful Our Search for Fractional Charge Particles}

This has been an upbeat talk about great discoveries and enormous progress in fundamental physics. But I must remind you that not all experiments are fruitful, in fact most experiments lead to small additions of knowledge or fail. An example 
is the dozen year search by Eric Lee and myself for isolatable, fractional charge, particles. All known isolatable particles such as the leptons have either zero charge or a magnitude of charge equal to the electron charge $q_{e}$. But there is no known law of matter that requires this to be true, it is simply an experimental observation. Therefore why not search for a particle with fractional charge, with for example a charge such as $1 / 2 \times q_{e}$ or $\sqrt{3} \times q_{e}$ or $\pi \times q_{e}$ ? Recall that quarks have fractional charge $2 / 3 \times q_{e}$ or $1 / 3 \times q_{e}$, but no one has succeeded in isolating quarks.

The experimental idea for the search came from collaborators Dinesh Loomba, Charles Hendricks, Klaus Lackner and Gordon Shaw; and we worked with wonderful graduate students Nancy Mar, Valerie Halyo and Irwin Lee. It is a beautiful apparatus, Fig. 11, a modern technology version of the Millikan oil drop experiment, in which very small drops are produced automatically and the drop charge is measured with a precision of about $q_{e} / 20$.

We have searched through a fraction of a gram of various materials but found no evidence for fractional charge particles. The alternative conclusions from this null result are:

- Fractional charge particles do not exist.

- Fractional charge particles exist but are too rare for us to detect in a fraction of a gram of material.

- Fractional charge particles exist but are not present in the matter through which we searched.

It would be wonderful to be able to search through tens of grams or a kilogram of matter but we do not know how to do this. Therefore we are stopping the search. If the search for fractional charge particles is a good idea then it will be taken up in the future by younger people with a better search method, if it is a bad idea then it should be abandoned. In either case it was a most enjoyable experiment, using areas of physics and chemistry of which I knew little before starting the search: fluid mechanics, colloidal suspensions, behavior of ions in a gas, statistical methods for analyzing millions of drop charge measurements.

\section{Dark Matter, A Change of Research Technology, and a New Telescope}

\subsection{A change in research technology}

About five years ago I became interested in doing research on the nature of Dark Matter. The known terrestrial search methods, Subsec. 6.4, did not interest me because of the uncertainty of the success of these methods. But the Dark Matter in the heavens existed and was already being studied and so I decided to go into astronomical research. This was a very large change in research technology for me. As a graduate student I had done research in atomic physics, then for 45 years my research was in particle accelerator physics, and there was the fractional charge search using drop physics, but I had never worked in astronomy. Nevertheless I 
jumped into astronomical research by joining a collaboration of about a dozen institutions who were proposing to build a large telescope to study Dark Matter as well as other astronomical phenomena, the Large Synoptic Survey Telescope(LSST). This jumping into new technologies with negligible preparation is a method I have followed throughout my life, in my early years as a chemical engineer and then as an experimental physicist for fifty years. Once the jump is made I learn what I need through the work itself or from colleagues or from books and articles. There are some bad times, but these times are short, and the advantage of this jumping is that I can learn fast.

\subsection{Detection of Dark Matter through gravitational bending of light}

Einstein's general relativity says that a light ray is deflected as passes near or through matter, Fig. 12. The existence of Dark Matter was established after Einstein's death, but his theory of general relativity is so encompassing that it applies to both Luminous Matter and Dark Matter. Light rays are deflected by both Luminous and Dark Matter. The observational consequence is the distortion of the telescope image of a distant luminous object such as a quasar or a galaxy, Fig. 13. For example, a galaxy that would be imaged directly as a circular disc might appear as an elliptical disc if the bundle of light rays passes near or through matter. An-

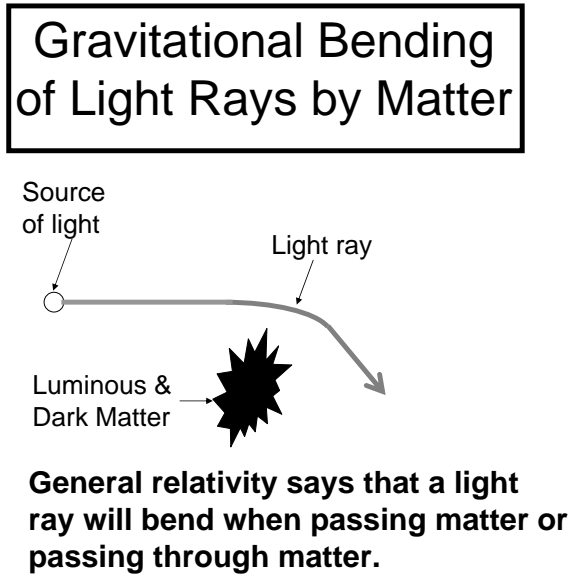

(Classical gravitational mechanics also predicts this but with an error of a factor of 2.)

Fig. 12. A light ray is bent as it passes near or though matter, whether the matter is Dark or Luminous. 
other possibility is that gravitational bending of the light ray bundle can produce two telescopic images of the same object, Fig. 14.

\section{Gravitational Bending of Light Rays by Matter}

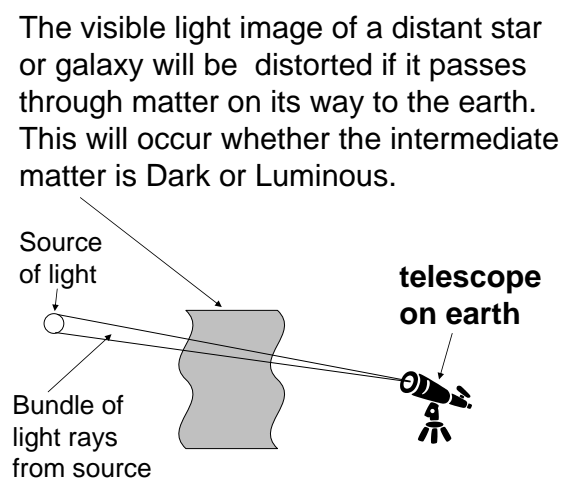

Fig. 13. The visible light image of a distant star or galaxy received by a telescope on earth is distorted as it passes near or though matter, whether the matter is Dark or Luminous.

\subsection{The Large Synoptic Survey Telescope(LSST)}

The LSST has major support from the U.S. National Science Foundation, crucial support from private donors and we look forward to major support from the U.S. Department of Energy. It is to be built by a collaboration of about a dozen national laboratories and universities. The LSST design calls for it being placed in Chile on a high, dry plateau where observing the heavens will be efficient. It has a triple mirror system with the major mirror 8.5 meters in diameter. It will swing across the night sky moving every 15 seconds thus producing pictures of the visible universe, Fig. 15.

My laboratory, SLAC, and the Brookhaven National Laboratory have major responsibilities for building the image detection system, consisting of about $3 \times 10^{9}$ light detecting elements in a 64 centimeter circular focal plane. Most of my work is development and engineering on a few parts of the camera, for example, on a device to test the flatness of the focal plane. The plane must be flat to better than 10 microns to give a well focused image of the sky. A human hair is about 100 microns in diameter. 


\section{Double Image Produced by Gravitational Lensing}

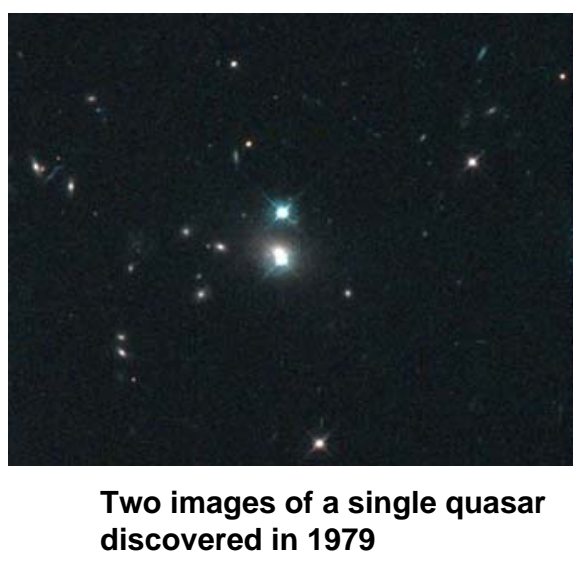

Fig. 14. A double image of a single quasar produced by gravitational bending of light.

Thus I am mostly an engineer again, but in a new technology. It is a pleasure to do this work. To me there is no difference between doing engineering and doing physics research as long as the problems are interesting to me. Of course I am also thinking about Dark Matter and what I can learn from observations of Dark Matter. Can I compete with all the younger researches in the field, many of whom have much more experience? I think so, I have years of experience in physics and my very ignorance in astrophysics means I am not yet overwhelmed by conventional ideas about Dark Matter.

\section{Old Unsolved Puzzles and New Questions in the 21st Century}

And so in the last hundred years we have learned a tremendous amount about elementary particles, fundamental forces, and cosmology. Yet we have many puzzles and questions. My generation may solve a few of these puzzles or answer a few of these questions, but we are self-brainwashed so that we think in fixed directions, so that we rarely conceive of new experimental and observation directions. But many of these puzzles and questions will be answered by the young women and men in this audience. They are as smart as my generation, they are better educated and they will have better experimental and observational technology. 


\section{Telescope swings back and forth viewing all of accessible sky}

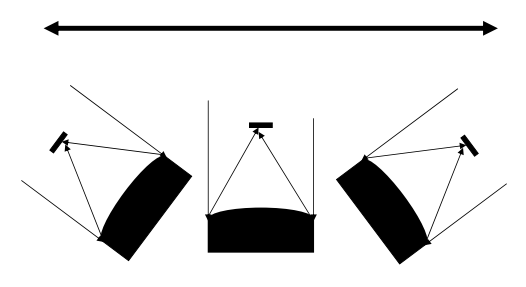

Fig. 15. The proposed Large Synoptic Survey Telescope(LSST).

\subsection{Continuing questions}

- What formula gives the masses of the six leptons and six quarks? We know many of these masses with precision but we do not know what to do with these numbers. What is the theory behind the formula? And what properties of elementary mass lead to a spread over at least 27 powers of 10, Fig. 16 ?

- Perhaps mass is not a fundamental property of a particle, but is a complicated, incidental property analogous to the surface color of a metal?

- What unified theory sets the properties of the four fundamental forces and the associated particles carrying the forces photon, gluon, W, Z, graviton? That is, what is the answer to Einstein's dream? Are there additional undiscovered forces?

- Are there more elementary particles beyond the leptons, quarks and force carrying particles? Heavier particles? Lighter particles? Fractional charge particles?

- What is the quantum mechanical nature of gravity? All past and present experiments and observations on the gravitational force concern the classical aspects of gravity such as general relativity. How can experiments and observations be carried out on the quantum nature of gravity? The experimental problem is that quantum mechanical experiments are mostly performed on small systems and then electromagnetic forces overwhelm the gravitational force. A few physicists believe that quantum mechanics cannot be applied to gravity.

- Are the leptons, quarks and force carrying particles composed of yet simpler 


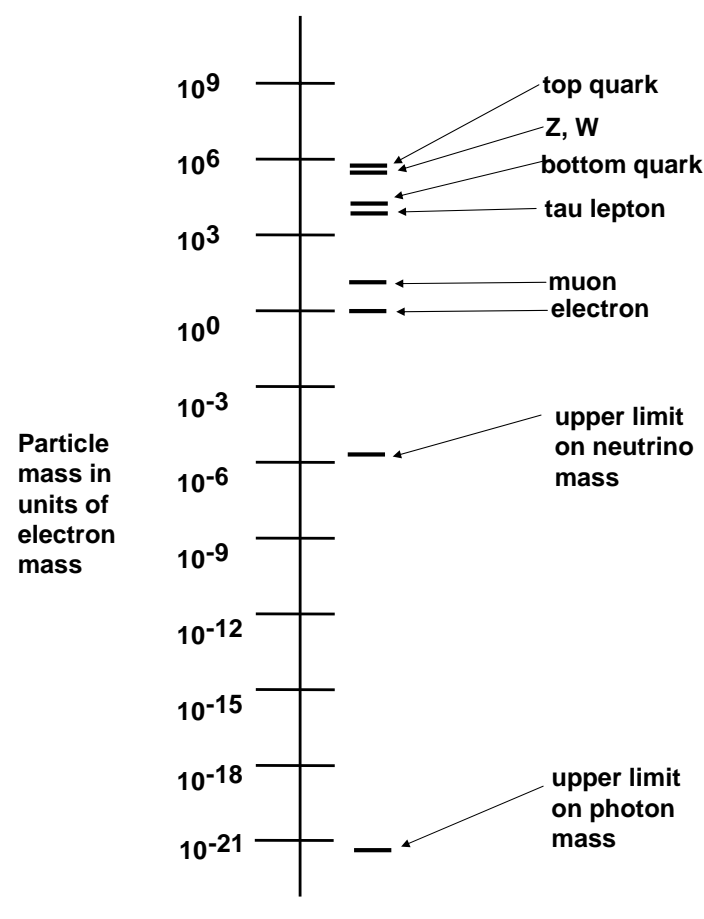

Fig. 16. The very large spread of masses of elementary particles. The masses are in units of the electron mass.

particles?

- What about the string theory models for elementary particles? Is one of the models true? The truth test is that the theory predicts phenomena not yet observed or that it allows the calculation of some fundamental numbers such as the charge or mass of the electron.

- If Dark Matter consists of one or more types of elementary particles, as most physicists and astronomers believe, what are the properties of the dark matter particle or particles? A few physicists and astronomers believe that the behavior of the gravitational force is not understood over large distances and that this is misinterpreted as Dark Matter. Even more speculative is it possible that Dark Matter does not consist of particles but is a new form of matter?

\subsection{Deeper questions}

- Are we asking the right questions?

- Is the human mind capable of deducing a complete, fundamental theory of matter 
and force?

\subsection{The crucial experimental question}

- Will physicists and astronomers find new ways in the 21st Century to do experiments and make observations that solve some of the puzzles and answer some questions that we are left with at the end of the 20th Century? I am confident that powerful, new research methods will be invented

\section{Acknowledgements}

I am very grateful to Professor Richard Gonsalves for the invitation to the Symposium and to give this public lecture. I tremendously enjoyed the hospitality of the Department of Physics and the opportunity to reminisce with Professor Lal Jain about older and simpler days in elementary particle physics.

This work was supported by Contract DE-AC03-76SFO0515 with the U. S. Department of Energy. 